\title{
Inteligencia emocional y conducta agresiva en el deporte ¿Puede inferir la modalidad deportivay las horas deentrenamiento? Emotional intelligence and aggressive behavior in sport. Can sports modality and hours of training infer? \\ *ManuelAntonio Gallardo Peña, *Marta Domíngez Escribano, **Carmen González González de Mesa *Universidad de Córdoba (España), **Universidad de Oviedo (España)
}

Resumen: El objetivo del presente estudio es comprobar si existe relación entre las horas semanales de entrenamiento, la inteligencia emocional (IE) y la agresividad que manifiestan los deportistas. También se pretende conocer si es sexo, edad, cantidad de horas de entrenamiento y modalidad de práctica (deportes individuales, de contacto o de no contacto), infieren en la inteligencia emocional y la agresividad. Los participantes en el estudio han sido 472 deportistas españoles ( $\mathrm{n}=235$ varones y $\mathrm{n}=237$ mujeres) pertenecientes a 22 clubs. Los resultados muestran que los deportistas que practican deportes de no contacto tienen mayor capacidad para identificar y valorar emociones que los deportistas que practican deportes de contacto; los practicantes de deportes de contacto, muestran mayores niveles de agresividad física o verbal, y hostilidad o ira, que los deportistas que practica deportes individuales y de no contacto. En cuanto al sexo los hombres presentan mayores niveles de agresividad física o verbal que las mujeres. Los de menos edad presentan mayores niveles de identificación y valoración emocional que los de media edad. Los de menor edad obtienen mayores puntuaciones que agresividad física o verbal y hostilidad e ira que los de mayor edad. Los sujetos que menos tiempo dedican a la práctica deportiva tienen mayores niveles de agresividad física o verbal que los que consumen más tiempo de entrenamiento. Los sujetos que tienen mayores niveles de agresividad física o verbal tienen menor capacidad para valorar las emociones.

Palabras clave: Agresividad, Inteligencia emocional, Deporte, Escala CIED-V2.0, Escala AQ.

Abstract: The objective of the present study is to verify if there is a relation between weekly hours of training, emotional intelligence (EI) and aggressiveness that athletes show. It is also intended to know if gender, age, number of hours of training and practice modality (individual sports, contact or non-contact) infer with emotional intelligence and aggression. The participants in the study were 472 Spanish athletes (n = 235 males and $n=237$ females) belonging to 22 clubs. The results show that athletes who practice non-contact sports have a greater capacity to identify and value emotions than athletes who practice contact sports; contact sports practitioners show higher levels of physical or verbal aggression and hostility or anger than athletes who practice individual and non-contact sports. Regarding gender, men have higher levels of physical or verbal aggression than women. Youngaged athletes have higher levels of identification and emotional assessment than those of middle age. Younger children obtain higher scores than physical or verbal aggressiveness and hostility and anger than older ones. Subjects who spend less time in sports have higher levels of physical or verbal aggression than those who spend more time training. Subjects who have higher levels of physical or verbal aggressiveness have less ability to assess emotions.

Keywords: Aggression, Emotional Intelligence, Sport, CIED-V2.0 questionnaire, AQ questionnaire.

\section{Introducción}

La violencia es un problema que, en la actualidad, es cada vez más preocupante debido al incremento que se ha producido. En diversos deportes el rendimiento de los jugadores y los equipos, parece verse influido por el grado de agresividad que se les exige (García García, Martínez y González-Gómez, 2017). Los comportamientos desadaptativos relacionados con la práctica deportiva son cada vez más frecuentes. El deporte se ha convertido en un hecho representativo de la sociedad actual (Sáenz, Gimeno, Gutiérrez, Lacambra, Arroyo del Bosque y Marcén, 2014) y puede facilitar el desarrollo de cualidades personales y sociales (Bredemeier y Shields, 1984). A pesar de que es una alternativa educativa muy beneficiosa para los menores, se dan actuaciones impropias por parte de jugadores, espectadores y entrenadores con una incidencia excesiva (Cox, 2008). En esta línea, estos autores indican que el deporte es un área de la interacción humana en el que la agresión algunas veces es rechazada, algunas veces condenada, pero otras muchas veces legitimada y tolerada (Sáenz et al., 2014).

El deporte, en origen, está considerado como una solución para atajar el problema de la agresividad (Monjas, Ponce y Gea, 2015). El uso de recursos que se desarrollen a través de juegos lúdicos y colaborativos que ayuden a los alumnos a vivenciar diferencias y conflictos grupales y que pongan a prueba la relación y tolerancia con los demás para presentar los verdaderos escenarios en los que se desarrolla la vida en sociedad (Pinheiro, Camerino y Sequeira, 2013).

Existe una multitud de estudios que demuestran que las conductas agresivas han llegado a ser demasiado habituales en las gradas, en los banquillos y sobre todo, en los terrenos de juego (Blasco y Orgilés, 2014). También se han realizado estudios en función de la modalidad deportiva. Bara, Scipião y Guillen (2004) trataron de buscar las seme-

Fecha recepción: 18-06-18. Fecha de aceptación: 16-07-18 Carmina González González de Mesa gmcarmen@uniovi.es janzas y diferencias entre los perfiles de personalidad de deportistas brasileños. Se encontraron diferencias significativas en los niveles de agresividad y también se encontraron diferencias más específicas entre los individuos de deportes de contacto directo físico y los que no tienen contacto físico. En función del género, en la población general, Sandoval (2001) llevó a cabo una investigación en la que comprobó que los hombres son más agresivos que las mujeres y éstas son más prosociales que los hombres, sin embargo otros autores (Baños, Ortiz-Camacho, Baena-Extremera y Zamarripa, 2018; Inglés, Torregrosa, GarcíaFernández, Martínez-Monteagudo, Estévez y Delgado, 2014) demuestran que las mujeres son más dadas a meterse en peleas que los varones. En cuanto al contexto deportivo, Blasco y Orgilés (2014) encontraron diferencias significativas en la agresividad física, más concretamente, las mujeres puntuaron más alto que los hombres en agresividad física. La agresividad, en sus diferentes manifestaciones verbales, físicas y frías o más sutil, la ira y la inestabilidad emocional, estimulan las conductas antisociales (Mestre, Samper, Tur-Porcar, Richaud y Mesurado, 2012).

La importancia de la Inteligencia Emocional (en adelante IE), radica en aprender a administrar las emociones para que éstas trabajen a favor de la persona. La investigación ha evidenciado que la carencia de IE, incide en un desempeño pobre de aspectos como problemas sociales, agresividad, depresión, etc. (Garaigordobil y Oñederra, 2010). En la misma línea, Petrides, Sangareau, Furnhan y Frederickson (2006) analizaron si las diferencias individuales en inteligencia emocional estaban relacionadas con la percepción que tenían los niños con sus compañeros. En concreto, este estudio analizó la inteligencia emocional rasgo y siete descriptores conductuales distintos en estudiantes de educación primaria: cooperación, disrupción, timidez, agresión, dependencia, liderazgo e intimidación. La evaluación de las variables conductuales se llevó a cabo a través de las nominaciones que los iguales y los profesores realizaron de todos los niños, utilizando estos siete descriptores conductuales. Los resultados indicaron que los niños con altas puntuaciones en inteligencia emocional rasgo recibieron más nominaciones de cooperación y liderazgo y un menor número de nominaciones de 
disrupción, agresióny dependencia. Además también se examinaron las nominaciones de los profesores mediante un análisis factorial revelando la existencia de dos factores ortogonales que abarcaron descripciones prosociales y antisociales. Así, los niños con alta inteligencia emocional puntuaron más alto en el factor prosocial y más bajo en el factor antisocial. Otros autores, que estudiaron la inteligencia emocional en deportistas adultos, no encontraron que el sexo pueda ser un elemento de inferencia, pero si la edad, los mayores los que gestionan mejor las emociones (Castro-Sánchez, Zurita-Ortega y Chacón-Cuberos, 2018).

El concepto de Inteligencia Emocional hace referenciaala capacidad de las personas para integrar eficazmente aspectos emocionales y cognitivos (Salovey y Grewal, 2005). En el contexto deportivo, la IE constituye un conjunto de meta-habilidades presentes en el deportista, que le capacitan para extraer información de las situaciones de competición y entrenamiento, de cara a percibir, controlar y emplear dicha información para maximizar su rendimiento personal (Arruza, GonzálezRodríguez, Palacios-Moreno,Arribas-Galarraga y Telletxea-Artzamendi, 2013). Muchos estudios defienden el efecto positivo que tiene el ejercicio físico sobre el bienestar psicológico, mental y el funcionamiento físico (Cadwell, Harrison, Adams, Quin y Greeson, 2010; Shuk-Fong, Hsiu-Hua y Chang Ning, 2009; Solanki y Lane, 2010). En la misma línea, Solanki y Lane (2010) estudiaron en una muestra de 315 estudiantes la relación entre la percepción de inteligencia emocional y las creencias sobre si el ejercicio permite el enriquecimiento de los estados anímicos. Para ello, correlacionó de forma significativa la inteligencia emocional con las creencias en relación a que el ejercicio podía ser utilizado para regular el estado anímico. Así, las personas que se ejercitaban y reportaban que el ejercicio físico les ayudó a regular sus estados de ánimo también obtuvieron mayores puntuaciones en inteligencia emocional. También indica Castro (2018) que los deportes de mayor contacto físico provocan en los sujetos mayor índice de violencia y menor autogestión de las emociones.

El conocimiento de uno mismo, la autorregulación de emociones, la automotivación, las habilidades sociales y la empatía son herramientas que todo deportista maneja en mayor o menor medida, bien sea consciente o inconscientemente (Ros, Moya-Faz y Garcés de los Fayos, 2013).

El objetivo de este trabajo es percibir si existe o no relación entre las horas semanales de entrenamiento, la inteligencia emocional y la agresividad que manifiestan los deportistas. También se pretende conocer si es sexo, edad, cantidad de horas de entrenamiento y modalidad de práctica, infieren en la inteligencia emocional y la agresividad.

\section{Metodología}

\section{Muestra}

Los participantes han sido 472 deportistas, $n=235$ varones y $n=$ 237 mujeres, que practican deportes de contacto medio-alto entre compañeros y contra adversarios, deportes sin contacto con adversarios y deportes individuales, con una media de edad de 17,53 años. Han participado en el estudio 22 clubs.

\section{Instrumento}

El instrumento utilizado para medir la inteligencia emocional ha sido el cuestionario CIED- V2.0, elaborado por González (2008) y basado en modelo de Mayer y Salovey de 1990. Está dividido en 3 factores: control y regulación emocional, Identificación y Valoración de las Emociones y aplicación y utilización de las emociones. Este instrumento es un autoinforme que consta de 25 ítems basado en una escala tipo Likert de 5 puntos siendo 1 Muy en desacuerdo a 5 Totalmente de acuerdo.

Para medir la agresividad se usó la adaptación española del Cuestionario de Agresividad de Buss y Perry (1992) adaptado por Andreu, Peña y Graña (2002) es un instrumento ampliamente utilizado tanto a nivel nacional como internacional. Está compuesto por 29 ítems que hacen relación a conductas y sentimientos agresivos. Estos 29 ítems están codificados con una escala tipo Likert de cinco puntos (1: comple- tamente falso para mí; 2: bastante falso para mí; 3: ni verdadero ni falso; 4: bastante verdadero para mí; 5: completamente verdadero para mí) y se estructuran en cuatro subescalas denominadas: agresividad física, compuesta por nueve ítems, agresividad verbal, compuesta por cinco ítems, ira, compuesta por siete ítems y, finalmente, hostilidad, compuesta por ocho ítems.

\section{Procedimiento}

El procedimiento seguido para solicitar la colaboración de diferentes clubs, una vez obtenido el permiso del comité de ética de la Universidad, se ha realizado en dos fases. Primeramente se envió a los clubs una carta explicativa de los objetivos del estudio, y posteriormente, mediante llamada telefónica, confirmar y gestionar la participación. Se seleccionaron 5 deportes y 24 clubs. Para la selección se ha procurado que los deportes abarcaran tres modalidades, contacto medio-alto (rugby y baloncesto), equipo pero sin contacto con los adversarios (voleibol) e individuales (natación y gimnasia rítmica).

Por último, una persona formada a tal efecto, ha sido la encargada de la distribución de las escalas. Han cumplimentado el cuestionario en sesión única de aproximadamente 10 minutos, explicándoles previamente que la participación era voluntaria, advirtiéndoles la importancia que tenía la cumplimentación de todos los ítems y garantizándoles el anonimato.

\section{Análisis estadístico}

Para comprobar la adecuación y fiabilidad de los cuestionarios se ha realizado un análisis descriptivo de medidas de tendencia central y dispersión de todos los ítems del cuestionario, un análisis factorial exploratorio (AFE) y un análisis de fiabilidad de los factores resultantes delAFE.

Para la investigación empírica se han aplicado análisis multivariado de la varianza y correlaciones bivariadas.

Los programas informáticos utilizados han sido el programa FACTOR 10, para el AFE, el cual ayuda a determinar el número de factores, y para ello se ha utilizado el método de Implementación Óptima de Análisis Paralelos, que Timmerman y Lorenzo (2011) lo aconsejan para este propósito, mediante 10.000 remuestreos. Este análisis se ha efectuado a partir de las correlaciones policóricas entre los ítems. La distribución de los factores se ha ejecutado por medio de mínimos cuadrados no ponderados aplicando el método Promin de rotación (Ferrando y Lorenzo, 2014)

El paquete estadístico SPSS 22 para Windows se ha utilizado para el análisis descriptivo (Medias, Desviación típica, Asimetría, Curtosos e índice de Homogeneidad corregido), Análisis de fiabilidad (Alfa de Crombch), análisis de homogeneidad (prueba de Levene), inferencial (MANOVAS) y correlaciones Bivariadas (Coeficiente de correlación de Pearson).

También se ha usado el programa G*Power para comprobar el tamaño del efecto ( $d$ de Choen).

\section{Resultados}

Se presentaran primeramente los resultados del AFE de la escala inteligencia emocional, CIED-V2.0.

Comenzando con el análisis descriptivo de los ítems, que indica buena distribución muestral al mostrar una Asimetría y una Curtosis con puntuaciones inferiores a 2,0 y un Índice de Homogeneidad corregida suficientemente alto.

Los resultados del AFE, obtenidos después de la rotación de los ítems, han mostrado tres factores. Los valores del estadístico de Bartlett [5707.1 (df = 300; P = .000010)] y el test de Kaiser-Meyer-Olkin $(\mathrm{KMO}=.904)$ muestran que la adecuación de los datos es buena para para ser sometidos al análisis factorial, garantizando el $54 \%$ de la varianza total. El índice de bondad de ajuste (GFI = .99) y la raíz cuadrática media de los residuales ( $\mathrm{RMSR}=.05$ ), indica muy buen ajuste de la estructura en tres dimensiones (García-Cueto, Gayo-Álvaro y Miranda-García, 998). 
Tabla 1.

Estadísticos descriptivos, matriz de componentes rotados y análisis de fiabilidad del cuestionario CIED-V2.0

\begin{tabular}{|c|c|c|c|c|c|c|c|c|}
\hline & $\mathrm{M}$ & DT. & Asi. & Cur. & IHc & F1 & F2 & F3 \\
\hline 1 Soy consciente de mis emociones cuando compito & 4.18 & 899 & -1.163 & 1.317 & .520 & & .386 & .377 \\
\hline $\begin{array}{l}2 \text { Si durante el transcurso de la competición siento una emoción positiva, sé cómo hacer que } \\
\text { perdure para que me vaya bien }\end{array}$ & 4.02 & 1.006 & -.993 & .573 & .592 & & & .491 \\
\hline $\begin{array}{l}3 \text { Reconozco las emociones que los/as adversarios/as están sintiendo mirando las expresiones, } \\
\text { tanto las faciales como corporales }\end{array}$ & 3.77 & 1.015 & -.728 & .249 & 498 & & .739 & \\
\hline 4 Cuando me enfrento a situaciones adversas, recuerdo similares situaciones que he superado & 3.90 & .998 & -.825 & 311 & .517 & .656 & & \\
\hline $\begin{array}{l}5 \text { Aunque la situación de la competición sea adversa, soy capaz de controlar los pensamientos } \\
\text { negativos y afrontar positivamente la competición }\end{array}$ & 3.63 & 1.003 & -.440 & -.385 & 539 & & & .677 \\
\hline 6 Ante una competición, casi siempre sé exactamente como me siento & 3.90 & .983 & -.873 & .492 & .589 & & & .601 \\
\hline 7 Cuando estoy de buen humor resuelvo las situaciones de la competición fácilmente & 4.21 & .875 & -1.071 & 1.094 & .523 & & .513 & \\
\hline 8 Durante la competición soy consciente de los mensajes no verbales que envían los/as demás & 3.94 & 1.021 & -.900 & .406 & .446 & & .854 & \\
\hline 9 A los/as compañeros/as les resulta fácil confiar en mí & 4.12 & .918 & -.990 & .701 & .422 & & .581 & \\
\hline 10 Ante una competición sé por qué cambia mi estado de ánimo & 3.92 & .965 & -.883 & 812 & .406 & & .522 & \\
\hline 11 Me automotivo imaginando un buen desenlace de las tareas que emprendo & 4.19 & .956 & -1.164 & 1.015 & .432 & .326 & & \\
\hline 12 Cuando me enfrento a una competición controlo mis emociones & 3.60 & 1.074 & -.535 & -.303 & .483 & & -.331 & .900 \\
\hline $\begin{array}{l}13 \text { Me es fácil reconocer mis emociones tanto en el entrenamiento como en la competición, } \\
\text { cuando las estoy sintiendo } \\
14 \text { En una competición intento tener buenos pensamientos y sentimientos y no preocuparme de }\end{array}$ & 4.01 & .918 & -.823 & .415 & .537 & & & 491 \\
\hline lo mal que me siento & 4.00 & .984 & -.949 & .576 & .522 & & & .622 \\
\hline 15 Cuando tengo que rendir bajo presión me siento seguro/a & 3.48 & 1.180 & -.467 & -.625 & .497 & & & .578 \\
\hline $\begin{array}{l}16 \text { Utilizo el sentido del humor para ayudarme a perseverar ante las adversidades que se dan en } \\
\text { el entrenamiento o competición }\end{array}$ & 4.03 & 1.001 & -1.004 & .579 & .395 & & & \\
\hline 17 Soy consciente de mis emociones cuando compito & 4.01 & .939 & -.788 & .208 & .632 & & & .699 \\
\hline $\begin{array}{l}18 \text { Si durante el transcurso de la competición siento una emoción positiva, sé cómo hacer que } \\
\text { perdure para que me vaya bien } \\
19 \text { Reconozco las emociones que los adversarios están sintiendo mirando las expresiones, tanto }\end{array}$ & 3.99 & .941 & -.801 & .243 & .676 & & & .441 \\
\hline las faciales como las corporales & 4.00 & .939 & -.762 & .165 & .520 & .976 & & \\
\hline 20 Cuando me enfrento a situaciones adversas recuerdo similares situaciones que he superado & 4.02 & .946 & -.812 & .274 & .592 & 1.037 & & \\
\hline $\begin{array}{l}\text { 11 Aunque la situacion de la competicion sea adversa, soy capaz de controlar los pensamientos } \\
\text { negativos y afrontar positivamente la competición }\end{array}$ & 3.76 & .991 & -.579 & -.130 & .498 & & & .908 \\
\hline 22 Ante una competición, casi siempre sé exactamente como me siento & 3.92 & .944 & -.739 & .268 & .517 & & & .671 \\
\hline 23 Cuando estoy de buen humor resuelvo las situaciones de la competición fácilmente & 4.13 & .893 & -1.002 & .913 & .539 & & .407 & \\
\hline 24 Durante la competición soy consciente de los mensajes no verbales que envían los/as demás & 3.90 & .953 & -.677 & -.011 & .589 & & .829 & \\
\hline 25 A los/as compañeros/as les es fácil confiar en mí & 4.00 & .941 & -.803 & .502 & .523 & & .490 & \\
\hline
\end{tabular}

Los factores resultantes se corresponden Control y Regulación Emocional (F1), Identificación y Valoración de las Emociones (F2) y Aplicación y Utilización Emocional (F3). Estos tres factores presentan un alfa de Cronbach superior a .700. Ver tabla 1.

Seguidamente se realizará el AFE de la escala de Agresividad en el Deporte, AQ, pero previamente se han reformulado los ítems que estaban formulados a la inversa. Observando los resultados obtenidos en el análisis descriptivo, se ha prescindido de las variables 15 y 24 por presentar un valor excesivamente bajo en el Índice de Homogeneidad corregido, y no han sido utilizados para el AFE. El resto de ítem muestra buen índice y distribución.

Realizada la rotación de todos los ítems resultantes, se concluye con dos factores, teniendo que prescindir también del ítem $n^{\circ} 10$, al cargar en los dos a la vez, con una diferencia menor de .100. El estadístico de Bartlett [5371.1 (df = 351; $\mathrm{P}=.000010)]$ y el test de KaiserMeyer-Olkin (KMO = .940) presentan una buena adecuación de los datos para ser sometidos a análisis factorial, confirmando el $45 \%$ de la varianza total. Elíndice de bondad de ajuste GFI = .98 y la raíz cuadrática media de los residuales RMSR $=.05$, indican un muy buen ajuste de la estructura en tres dimensiones (García-Cueto et al. 1998).

Los factores resultantes se corresponden con Hostilidad o Ira (F1) yAgresividad Física o Verbal(F2), ambos muestran un Alfa deCrombach superior a .700. Ver tabla 2.

Dicho esto, se procede a presentar los resultados del análisis multivariado de la varianza (MANOVA) con el objeto de conocer la inferencia de las variables independientes sobre las variables dependientes.

Tomando como variables independientes el Sexo y la Modalidad Deportiva (2x3) y como variables dependientes los tres factores resultantes del cuestionario de Inteligencia Emocional CIED-V2.0 (Cont.Regul.Emoc, Ident.Valor.Emoc y Aplic.Utiliz.Emoc). Emergió un efecto significativo multivariado para la Modalidad Deportiva ( $.959, \mathrm{~F}_{(6,928)}=3.270, p<.05, c^{2}=.021,1$-â $\left.=.934\right)$, pero no así para el Sexo; también surgió un efecto significativo para la interacción Sexo*Modalidad Deportiva $\left(\check{Z}=.940, \mathrm{~F}_{(6,928)}=4.869, p<.001\right.$, $c^{2}=$ .031, 1-â = .992).

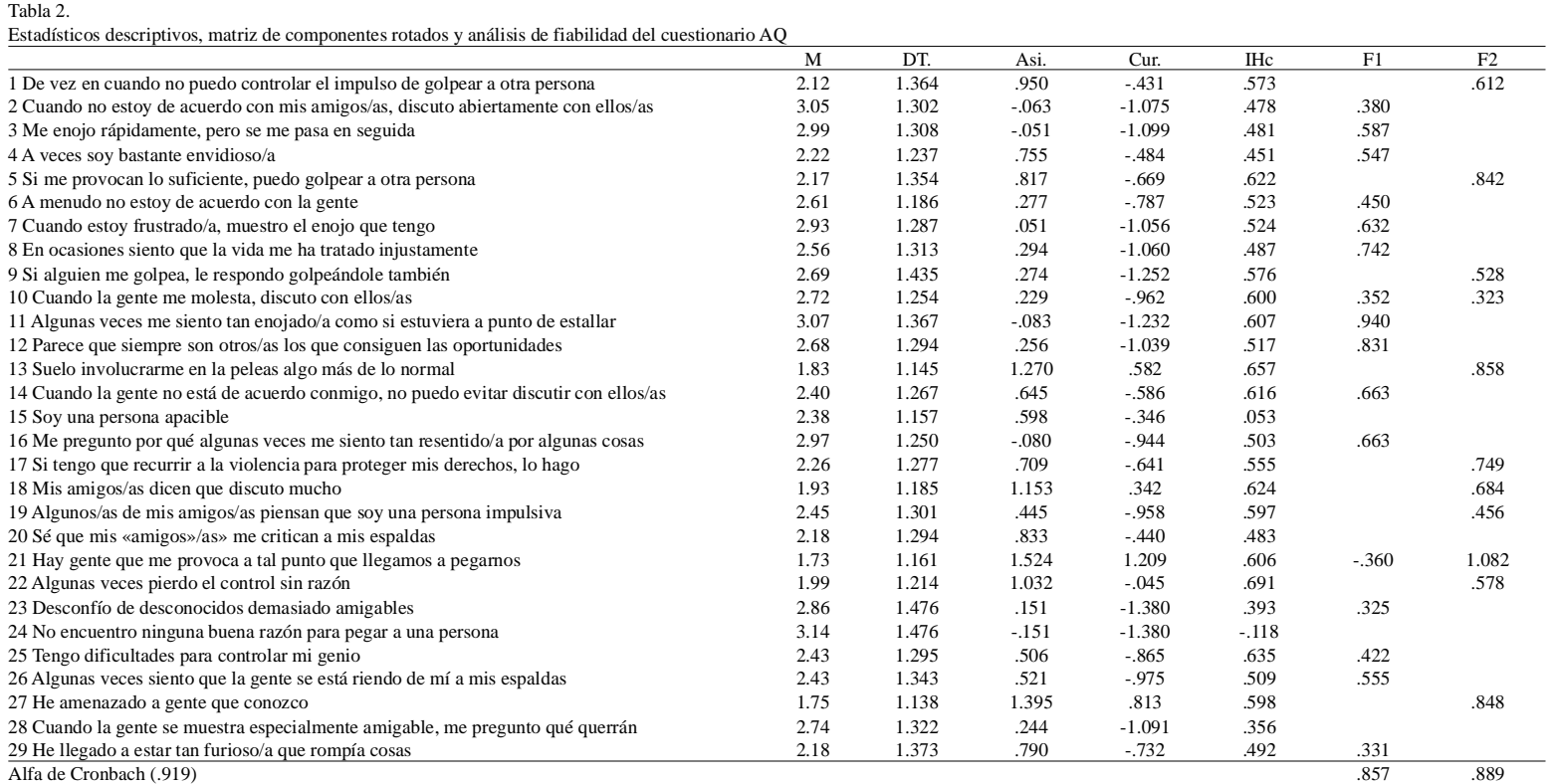


Los posteriores análisis univariados han revelado que con respecto a la modalidad deportiva presenta valores estadísticamente significativos en el factor Ident.Valor.Emoc $\left(\mathrm{F}_{(1,471)}=4.161, p<.05\right.$, $\mathrm{c}^{2}=.018,1$ â = .733), puntuando más alto los que practican deportes de No Contacto que los de Contacto. (DM = .219 y tamaño del efecto bajo, $d=$ .36). Ver tabla 4.

Seguidamente se realizó un MANOVA para analizar la Agresividad, tomando también como variables independientes el Sexo y la Modalidad Deportiva (2x3) y como variables dependientes los dos factores resultantes (Host.Ira y Agr.Fis.Verb). Surgió un efecto significativo multivariado para el Sexo $\left(\check{Z}=.977, \mathrm{~F}_{(2,465)}=5.525, p<.005, c^{2}=.023\right.$, 1 -â $=.852)$, y para Modalidad Deportiva $\left(\check{Z}=.965, \mathrm{~F}_{(4,930)}=4,133\right.$, $\mathrm{p}$ $<.005, c^{2}=.017,1$-â $\left.=.920\right)$, pero no así en la intersección Sexo*Modalidad Deportiva.

En el análisis univariado, en cuanto al sexo, han emergido diferencias estadísticamente significativas en el factor Agr.Fis.Verb, puntuando más alto los hombres $\left(\mathrm{F}_{(1,471)}=7.526, p<.05, c^{2}=.002\right.$, 1-â $\left.=.782\right)$, $\mathrm{DM}=.259$ y con tamaño del efecto medio, $(d=.44)$. Se han podido observar que los deportistas que practican deportes de Contacto puntúan más alto que los de No Contacto (DM = .244) en el factor Hostilidad o Ira $\left(\mathrm{F}_{(2,470)}=3.071, p<.05\right.$, ç² = .013, 1-â = .592); también puntúan más alto los deportes de Contacto que los deportes de No Contacto $(\mathrm{DM}=.328, d=.60)$ y que los Individuales ( $\mathrm{DM}=.271, d=.46)$, en el factor Agresividad física o verbal, presentando ambos un tamaño del efecto medio, $\left(\mathrm{F}_{(2,470)}=6.009, p<.05, c^{2}=.025\right.$, 1-â $\left.=.882\right)$.

También se realizó una MANOVA(4X3) tomando como variables independientes los grupos de Edad (14 años o menos, de 15 a 18, de 19 a 24 y 25 o más) y la Cantidad de Entrenamiento Semanales (menos de $5 \mathrm{~h} / \mathrm{sem}$., entre 5 y $8 \mathrm{~h} / \mathrm{sem}$. y más de $8 \mathrm{~h} / \mathrm{sem}$.) y como variables dependientes los tres factores resultantes del cuestionario de Inteligencia Emocional CIED-V2.0 (Cont.Regul.Emoc, Ident.Valor.Emoc y Aplic.Utiliz.Emoc). Ha manado efectos significativo multivariado para los grupos de Edad $\left(\check{Z}=.950, \mathrm{~F}_{(9,1115)}=2.616, p<.005, c^{2}=.017,1\right.$ $\hat{\mathrm{a}}=$.883). No se hallaron efectos estadísticamente significativos para la Cantidad de Deporte, ni para la intersección Edad*Cantidad de Deporte.

Los posteriores análisis univariados han revelado que los grupos de edad presentan valores estadísticamente significativos en Ident.Valor.Emoc $\left(\mathrm{F}_{(3,469)}=3.584, p<.014, c^{2}=.023\right.$, 1-â $\left.=.790\right)$ puntuando más alto menores de 14 años que los sujetos que tienen entre 19 y 24 años, $\mathrm{DM}=.250, \mathrm{~d}=.46$; en Aplic.Utiliz.Emoc $\left(\mathrm{F}_{(3,469)}=\right.$ $6.228, p<.001, c^{2}=.039,1$-â $=.964$ ), puntuando más alto los menores de 14 años que los sujetos entre $15-18$ años ( $\mathrm{DM}=.276, d=.37$ ), que los de 19- 24 años ( $\mathrm{DM}=.273, d=.48$ ), y que de más de 25 años $(\mathrm{DM}=.321, d=.40)$

Se realizó un MANOVA tomando como variables independientes el grupo de Edad y Cantidad de Entrenamiento Semanal (4x3) y como variables dependientes los dos factores resultantes del cuestionario de AgresividadAQ(Host.Ira y Agr.Fis.Verb). Emergió un efecto significativo multivariado para la intersección entre grupo de Edad*Cantidad de Entrenamiento Semanal $\left(\breve{Z}=.951, \mathrm{~F}_{(12,918)}=1.939, p<.05\right.$, ç ${ }^{2}=.025,1$ â $=$.918).

En cuanto al análisis univariado, en el factor Agr.Fis.Verb, en relación a Edad, puntúan más alto los menores de 14 años que los mayores de 25 años, $\mathrm{DM}=.431, d=.46$, $\left(\mathrm{F}_{(3,469)}=1.260, p<.05 c^{2}=.019,1\right.$ â = .702), y así mismo, los sujetos que dedican más de $8 \mathrm{~h} / \mathrm{sem}$ puntúan por debajo que los sujetos que dedican menos de $5 \mathrm{~h} / \mathrm{sem}, \mathrm{DM}=.36, d$ $=.36,\left(\mathrm{~F}_{(2,470)}=3.405, p<.05 c^{2}=.015,1-\hat{\mathrm{a}}=.640\right)$.

En las tablas 3 y 4 se presentan todos los datos expuestos en los párrafos anteriores.

Por último se han calculado las correlaciones entre los factores de Inteligencia Emocional y Agresividad. Como podemos observar el Cont.Regul.Emoc correlaciona positivamente con Ident.Valor.Emoc y Aplic.Utiliz.Emoc. El factor Ident. Valor.Emoc correlaciona positivamente con Aplic.Utiliz.Emoc. El factor Host.Ira correlaciona con Agr.Fis.Verb, y en sentido negativo Aplic.Utiliz.Emoc. Ver tabla 5.
Tabla

Resultados estadísticamente significativos del Análisis multivariado de la varianza (MANOVA)

\begin{tabular}{|c|c|c|c|c|c|c|c|c|c|c|}
\hline & \multicolumn{5}{|c|}{ Inteligencia emocional } & \multicolumn{5}{|c|}{ Agresividad } \\
\hline & ? & $F_{(G)}$ & Sig. & $?^{2}$ & $1-\beta$ & ? & $F_{(G)}$ & Sig. & $?^{2}$ & $1-\beta$ \\
\hline & & & & & & .977 & $5.525_{(2,465)}$ & .004 & .023 & .852 \\
\hline & .95 & 3.2 & .003 & 021 & .934 & .965 & $4.133_{(4,930)}$ & .003 & .017 & .92 \\
\hline $0 * 1$ & .94 & 4.869 & .000 & .031 & .992 & & & & & \\
\hline 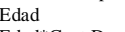 & .950 & $2.616_{(9,1115)}$ & .005 & 017 & .883 & & & & & \\
\hline d ${ }^{2}$ & & & & & & .915 & & .027 & 025 & .91 \\
\hline
\end{tabular}

Tabla 4.

Resultados estadísticamente significativos del análisis univariado, diferencias de medias y tamaño del efecto

\begin{tabular}{|c|c|c|c|c|c|c|c|c|}
\hline & & $F_{(\text {GI })}$ & $p$ & $?$ & $1-B$ & Variables & DM & $d$ \\
\hline Sexo & Agr.Fis.Verb & $7.526_{(1,471)}$ & .006 . & 016 & .782 & Varones - mujeres & .259 & 44 \\
\hline \multirow{3}{*}{ Mod.Dep } & Ident.Valor.Emoc & $4.161_{(1,471)}$ & .016 . & 018 & .733 & Contacto - no contacto & -.219 & .36 \\
\hline & Host.Ira & $3.071_{(2,470)}$ & 047 & 013 & .592 & Contacto - no contacto & .244 & .32 \\
\hline & Agr.Fis.Verb & $6.009_{(2,470)}$ & & 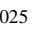 & .882 & $\begin{array}{l}\text { Contacto - no contacto } \\
\text { Contacto - individual }\end{array}$ & .328 & 60 \\
\hline \multirow{5}{*}{ Edad } & Ident.Valor.Emoc & $3.584_{(3,469)}$ & .014 . & 023 & .790 & 14 o menos -19 a 24 & .250 & .46 \\
\hline & & & & & & 14 o menos -15 a 18 & .276 & .37 \\
\hline & Aplic.Utiliz.Emoc & $6.228_{(3,469)}$ & .000 & 039 & .964 & 14 o menos - 19 a 24 & 273 & .48 \\
\hline & & & & & & 14 o menos -25 o más & 321 & 40 \\
\hline & Agr.Fis.Verb & $1.260_{c_{3}}$ & .032 & 019 & .702 & 14 o menos - 25 o más & .431 & .46 \\
\hline n.top & Agr.Fis.Verb & $3.405_{(2,}$ & .034 & 015 & .640 & Menos de 5 - más de 8 & 323 & 36 \\
\hline
\end{tabular}

Tabla

Correlaciones bivariadas entre factores

\begin{tabular}{lccccc}
\hline & 1 & 2 & 3 & 4 & 5 \\
\hline 1 Control y regulación emocional & 1 & $.444^{* *}$ & $.569^{*+*}$ & .006 & -.014 \\
2 Identificación y valoración emocional & & 1 & $.617^{*+*}$ & .003 & -.007 \\
3 Aplicación y valoración emocional & & & 1 & .006 & $-.142^{* *}$ \\
4 Agresividad física o verbal & & & & 1 & $.618^{*+*}$ \\
5 Hostilidad e Ira & & & & & \\
\hline **. & & & & &
\end{tabular}

5 Hostilidad e Ira

\section{Discusión}

Este estudio trata de conocer si existe relación entre la agresividad, la inteligencia emocional y las horas dedicadas semanalmente a entrenar, además de la inferencia que puede tener en dichos factores el sexo, edad y modalidad de práctica.

En cuanto a la Inteligencia Emociona, se ha comprobado que en el factor Identificación y Valoración de las Emociones otorgaron puntuaciones más altas los sujetos que practican deportes de no contacto que los que realizan deportes de contacto. Según Castro (2018) los sujetos que realizan deportes colectivos con contacto presentan una mayor falta de habilidades emocionales, siendo a su vez los que generan mayores niveles de violencia.

En los niveles de agresividad, tanto física como verbal, se ha encontrado que los hombres puntúan más alto que las mujeres. Esto está en contraposición a los resultados encontrados en el estudio de Inglés, Torregrosa, García-Fernández, Martínez-Monteagudo, Estévez y Delgado (2014) y Baños, Ortiz-Camacho, Baena-Extremera y Zamarripa (2018), que explican que las mujeres puntuaban por encima de los hombres a la hora de involucrarse en peleas o sentirse capaz de golpear a otros por algún motivo, así como presentar pensamientos negativos sobre los demás y sobre la propia vida, aspectos que están más relacionados con las dificultades para gestionar las emociones (propias y ajenas).

Por otro lado, en hostilidad o ira y en agresión física o verbal, los sujetos que practican deportes de contacto puntúan más alto que los practican deportes en los que no existe directamente contacto físico, tanto de equipo como individuales. Esto va en contraposición con los resultados encontrados en el estudio de Steyn y Roux (2009) donde la agresividad verbal y hostilidad obtuvieron puntuaciones significativamente más bajas en deportistas que participan en deportes de contacto.

Respecto a los niveles de identificación y valoración emocional en función de la edad, se ha podido encontrar en el presente estudio, que los sujetos menores de 14 años tienen mayor capacidad para valorar las emociones e identificarlas que los sujetos de 19- 24 años. En contraposición con los encontrados en Sainz (2010), Bekendam (2013) y Martín (2013)en los cuales se encontró que los sujetos de mayor edad puntúan más alto en la identificar y valorar las emociones que los sujetos de menor edad. Esto se puede justificar en base a que la inteligencia emocional mejora en función de la acumulación de experiencias vividas (Keefer, Holden y Parker, 2013). 
Cabe señalar que los deportistas menores de 14 años, en hostilidad o ira, conceden puntuaciones más altas que los mayores de 25 años, mientras que los sujetos de 15-18 años otorgan valoraciones superiores que los mayores de 25 años. De igual modo, en agresividad física o verbal, puntúan más alto los menores de 14 años que los mayores de 25 años. Esta afirmación está en contraposición con los resultados obtenidos en el estudio de Frognery Pilz (1982), donde defendían que a mayor edad, mayor agresividad en el deporte. Aunque esta afirmación es un poco contradictoria ya que la experiencia y la edad se caracterizan en general por proporcionar un mayor autocontrol.

En agresividad física o verbal, puntúan más alto los que entrenan pocas horas semanales que los sujetos que dedican mucho tiempo a la preparación. Aunque no se ha podido encontrar ningún estudio actual que relacione el tiempo de entrenamiento y la agresividad física o verbal, se puede justificar debido al dominio del ejercicio, ya que al dedicarle más horas, no solo se entrenan aspectos técnicos sino también psicológicos como el control de las emociones. Por tanto a menor número de horas, menor control dentro de dicho deporte.

En cuanto a la inteligencia emocional, se pudo observar que los sujetos que tienen mayor control sobre sus emociones y pueden regularlas, también son capaces de identificarlas, realizar mejores valoraciones y utilizarlas. También los sujetos que sabían identificar las emociones y valorar las mismas podían hacer uso de ellas de mejor forma. Estas afirmaciones van en línea con los resultados mostrados en el estudio de Salguero, Fernández-Berrocal, Ruiz-Aranda, Castilloy Palomera(2011) donde hallaron que una mayor habilidad para entender las emociones de otras personas tenían una mejor percepción de las mismas.

Los sujetos que mostraban mayor agresividad, tanto física como verbal, puntuaban más bajo en valoración de las emociones. Esta afirmación va en línea con el estudio realizado por Inglés et al. (2014) los cuales concluyeron que involucrarse en peleas o sentirse capaz de golpear a otros por algún motivo, así como presentar pensamientos negativos sobre los demás y sobre la vida, son los aspectos más relacionados con las dificultades de gestionar las emociones (propias y ajenas). Por último, se halló que los sujetos que muestran mayor agresividad física y verbal son los que tienen mayor hostilidad e ira.

\section{Conclusiones}

Se ha llegado a las siguientes conclusiones:

- Los deportistas que practican deportes de no contacto tienen mayor capacidad para identificar y valorar las emociones que los deportistas que practican deportes de contacto.

- Los hombres presentan mayores niveles en agresividad física o verbal que las mujeres.

- Los deportistas que practican deportes de contacto tienen mayores niveles de agresividad y hostilidad que los deportistas que practican deportes individuales y colectivos sin contacto.

En relación a la edad y las emociones, los menores de 14 años tienen mayor capacidad para identificar y valorar las emociones que los sujetos entre 19 y 24 años. En relación a la hostilidad y agresividad física, los deportistas menores de 14 años obtuvieron mayores puntuaciones en hostilidad o ira que los mayores de 25 años, mientras que los sujetos de 15-18 años otorgan valoraciones más altas que los mayores de 25 años. Los deportistas menores de 14 años puntúan más alto en los niveles de agresividad física o verbal que los sujetos mayores de 25 años.

Cuando se analizó las horas de entrenamiento se concluyó que los deportistas que dedican menos de $5 \mathrm{~h} / \mathrm{sem}$. puntúan más alto que agresividad física o verbal que los sujetos que dedican más de $8 \mathrm{~h} / \mathrm{sem}$.

Los deportistas que muestran mayor control sobre las sus emociones y regulan las mimas, también son capaces de identificarlas, realizando mejores valoraciones de las misma. Del mismo modo, los deportistas que pueden identificar las emociones y valorar las mismas también pueden hacer uso de las mismas de mejor forma.

- Los deportistas que tiene mayor agresividad, tanto física como verbal, tienen menor capacidad para valorar las emociones.

Teniendo en cuenta el objetivo planteado a principio de la investigación, y en base a los resultados obtenidos, se puede concluir que existe una relación entre inteligencia emocional y agresividad; con respecto al sexo, se ha encontrado diferencia estadísticamente significativa en agresividad; la edad influye tanto en inteligencia emocional como agresividad; las horas de práctica deportiva infieren en la agresividad; y la modalidad deportiva muestra diferencias en inteligencia emocional y agresividad.

\section{Referencias}

Andreu, J., Peña, M. y Graña, J. (2002). Adaptación psicométrica de la versión española del Cuestionario de Agresión. Psicothema, 14(2), 476-482.

Arruza, J. A., González-Rodríguez, O., Palacios-Moreno, M., Arribas- Galarraga, S. y TelletxeaArtzamendi, S. (2013). Un modelo de medida de la inteligencia emocional percibida en contextos deportivo/competitivos. Revista de Psicología del Deporte, 22(2), 405-413.

Baños, R, Ortiz-Camacho, M., Baena-Extremera, A y Zamarripa, J. (2018). Efecto del género del docente en la importancia de la Educación Física, clima motivacional, comportamientos disruptivos, la intención de práctica futura y redimiento académico. Retos, 33, 252-257.

Bara, M.G, Scipiao, L.C. y Guillen, F. (2004). La personalidad de deportistas brasileños de alto nivel: Comparación entre diferentes modalidades deportivas. Cuadernos de Psicología del Deporte, $4(1,2), 117-127$.

Bekendam, N. (2013). Diferencias en inteligencia, inteligencia emocional y personalidad entre nadadores y sujetos sedentarios. Tesis doctoral: Universidad Europea de Madrid.

Blasco, M., Orgilés, M. (2014). Agresividad en menores de 18 años jugadores de fútbol: Diferencias en función del sexo y la edad en comparación con los jugadores de baloncesto. Cuadernos de Psicología del deporte, 14(2), 21-26.

Bredemeier, B. y Shields, D. (1984). The utility of moral stage analysis in the investigation of athletic aggression. Sociology of Sport Journal, 1, 138-149.

Buss, A.H. y Perry, M. (1992). The aggression questionnaire. Journal of Personality and Social Psychology, 63, $452-459$

Cadwell, K., Harrison, M., Adams, M., Quin, R., y Greeson, J. (2010). Developing mindfulness in college students through movement-based courses: Effects on self regulatory, self efficacy, mood, stress, and sleep quality. Journal of American College Health, 58(5), 433-442.

Castro, M. (2018). Análisis de diversos factores psicológicos en diferentes modalidades deportivas en función del nivel competitivo. Tesis doctoral: Universidad de Jaén.

Castro-Sánchez, M.; Zurita-Ortega, F.; Chacón-Cuberos, R. (2018). Inteligencia emocional en deportistas en función del sexo, la edad y la modalidad deportiva practicada. Sportis Sci J, 4(1), 288305

DOI:https://doi.org/10.17979/sportis.2018.4.2.3296

Cox, R.H. (2008). Psicología del deporte: Conceptos y sus aplicaciones. Madrid: Editorial Médica Panamericana S.A

Ferrando, P. y Lorenzo, U. (2014). El análisis factorial exploratorio de los ítems: algunas consideraciones adicionales. Anales de Psicología, 30(3), 1170-1175.

Frogner, E. y Pilz, G. A. (1982). Untersuchung zur EÏnstellung von jugendlichen Fussballspielern und spielerinnen zu Regeln und Normen im Sport (Investigaciones de las actitudes de jóvenes jugadores y jugadoras de fútbol frente a normas y reglas). En G.Pilz (ed.), Sport und Gemalt (Deporte y violencia). Schorndorf: Verlag Kart Hofmann.

Garaigordobil, M. y Oñederra, J.A. (2010). Inteligencia emocional en las víctimas de acoso escolar $\mathrm{y}$ en los agresores. European Journal of Education and Psychology, 3, 243-254.

García-Cueto,E., Gayo-Álvaro, P. y Miranda-García, R. (1998). Bondad de ajuste en el análisis factorial confirmatorio. Psicothema, 10(3), 717-724.

García, P.A., Martínez, J.A. y González-Gómez, F.J. (2017). Influencia de la agresividad sobre el rendimiento de equipos de fútbol en España / The Influence of Aggressiveness on the Performance of Football Teams in Spain. Revista Internacional de Medicina y Ciencias de la Actividad Física y el Deporte vol. 17(66) pp. 317-334.

González, O. (2008). Análisis y validación de un cuestionario de inteligencia emocional en diferentes contextos deportivos. Tesis doctoral. País Vasco: Universidad del País Vasco.

Inglés, J., Torregrosa, M., García-Fernández, J., Martínez-Monteagudo, M., Estévez, E. y Delgado, B. (2014). Conducta agresiva e inteligencia emocional en la adolescencia. European Journal of Education and Psychology, 7(1), 29-41

Keefer, K. V., Holden, R. R. y Parker, J. D. A. (2013). Longitudinal Assessment of Trait Emotional Intelligence: Measurement Invariance and Construct Continuity From Late Childhood to Adolescence. Psychological Assessment, 4, 1255-1272.

Martín, M. (2013). Análisis de un modelo estructural de inteligencia emocional y motivación autodeterminada en el deporte. Tesis doctoral: Universidad de Valencia.

Mayer, J.D. y Salovey, P. (1997). What is emotional intelligence? En P. Salovey y D. Sluyter (Eds.), Emotional development and emotional intelligence, (pp. 3-31). Nueva York: Basic Books.

Mestre, V., Samper, P., Tur-Porcar, A. M., Richaud de Minzi, M. C. y Mesurado, B. (2012). Emociones, estilos de afrontamiento y agresividad en la adolescencia. Universitas Psychologica, 11(4), 1263-1275.

Monjas, R., Ponce, A. y Gea, J.M. (2015). La transmisión de valores a través del deporte. Deporte escolar y deporte federado: relaciones, puentes y posibles trasferencias. Retos, 28, 276-284.

Petrides, K.V., Sangareau, Y., Furnham, A. y Frederickson, N. (2006). Trait emotional intelligence and children's peer relations at school. Social Development, 15(3), 537-547.

Pinheiro, V., Camerino, O. y Sequeira, P. (2013). Recursos para potenciar el fair play en la iniciación deportiva. Retos. Nuevas tendencias en Educación Física, Deporte y Recreación, 24, 97-99.

Ros, A., Moya-Faz, F, Garcés de los Fayos, E. (2013). Inteligencia emocional y deporte: situación actual del estado de la investigación. Cuadernos de Psicología del Deporte, 13(1) 105-112.

. Evaluación de la violencia y deportivid Psicología del Deporte, 15(1), 211-222.

Sainz, M. (2010). Creatividad, personalidad y competencia socio-emocional en alumnos de alta habilidad versus no alta habilidad. Tesis doctoral: Universidad de Murcia.

Salguero, J.M., Fernández-Berrocal, P., Ruiz-Aranda, D., Castillo, R. y Palomera, R. (2011). Inteligencia emocional y ajuste psicosocial en la adolescencia: El papel de la percepción emocional. European Journal of Education and Psychology, 4(2), 143-152.

Salovey, P. y Grewal, D. (2005). The Science of emotional intelligence. Currents Directions in Psychological Science, 14, 281-285.

Sandoval, J. (2001). Ambiente escolar, familiar y comunitario en relación con los comportamientos agresivos y prosociales en niños de 3 a 12 años. Revista Facultad Nacional Salud Pública, 24, 30-39.

Shuk-Fong, L., Hsiu-Hua, W. y Chang Ning, L. (2009). The comparison of emotional intelligence in college students participating in different levels of exercise. Asian Journal of Physical Education \& Recreation, 15(1), 48-55

Solanki, D. y Lane, A. M. (2010). Relationships between exercise as a mood regulation strategy and trait emotional intelligence. Asian Journal of Sports Medicine, 1(4), 195-200.

Steyn, B. y Roux, S. (2009). Aggression and psychological well-being of adolescent takewondo partecipants in comparison with hockey partecipants and non-sport group. African Journal for Physical, Health Education, Recreation and Dance, 15(1), 32-43

Timmerman, M. y Lorenzo, U. (2011). Dimensionality assessment of ordered polytomous items with parallel analysis. Psychological Methods, 16(2), 2019-220.

Retos, número 35, 2019 (1ºmestre) 